\title{
The Chronic Traumatic Stress Framework: A conceptual model to guide empirical investigation of comprehensive treatments for refugees and survivors of torture
}

\author{
Karen Fondacaro*, Emily Mazzulla*
}

Key points of interest:

- A biopsychosocial framework outlining the physical and psychological impact of traumatic war events, post-migration living stress, and daily hassles in refugees and survivors of torture is proposed.

- We discuss the importance of measurement when implementing biopsychosocial multimodal interventions including the following domains: 1) supportive (community or group connection);

2) psychoeducation and symptom reduction; 3) addressing the trauma narrative; and/or 4) attention to physical health.

- The Chronic Traumatic Stress (CTS) Framework introduced in this article serves as a guide for empirical investigation of outcomes of interdisciplinary treatments beyond a focus on pathology such as that outlined in post-traumatic stress disorder (PTSD).

^) University of Vermont, New England Survivors of Torture and Trauma

Correspondence to: emily.mazzulla@uvm.edu

\begin{abstract}
An increasing number of refugees and survivors of torture resettled in the United States are presenting to clinics for treatment related to trauma and postmigration difficulties. Although clinicians experienced in treating trauma with diverse populations may recognize the limitations of a PTSD diagnosis, one of the primary diagnoses received by refugees and survivors of torture remains post-traumatic stress disorder (PTSD). A variety of interventions exist (e.g., supportive, trauma specific, interdisciplinary including physical, social and psychological) for survivors of torture and trauma that move beyond this diagnosis, however, a unifying conceptual model is needed to guide treatment and further the empirical investigation and evidence base in this growing field. In this paper, we propose a broader biopsychosocial framework of the impact of traumatic war events including the measurement of stress related to post migration living difficulties, and daily hassles while highlighting the importance of protective and risk factors. Intervention outcomes emphasize resilience, physical well-being, and mental well-being, along with traumatic stress symptoms. We describe Chronic Traumatic Stress (CTS) as an integrated and unifying framework which provides guidance for the growing number of providers conducting assessment and
\end{abstract}


intervention with refugees and survivors of torture. We also highlight that this model is specifically designed for empirical testing.

Keywords: torture, refugees, PTSD, trauma

An overwhelming 65.6 million individuals have been displaced due to war conflict, persecution, and/or unstable political infrastructure contributing to record numbers worldwide (UNHCR, 2018). These refugees, asylum seekers and internally displaced persons often endure severe hardships including torture and other traumatic events (Campbell, 2007); (Porter \& Haslam, 2005). Many refugees experience chronic traumatic stressors throughout war, during flight and the resettlement process. Additionally, post migration living difficulties and daily stress have been shown to exacerbate the impact of war related trauma (Miller \& Rasmussen, 2010). The complex physical and mental health needs of the growing numbers of resettled refugees often challenge the professional bounds of clinicians and mental health providers. Mental and physical health concerns can be far reaching, affecting extended families and communities within which these individuals are attempting to rebuild their lives. While one of the most commonly studied Western diagnoses is post-traumatic stress disorder (PTSD) (Fazel, Wheeler, \& Danesh, 2005; Steel et al., 2009; Weiss et al., 2016), the applicability of assigning traditional, western diagnoses to refugees and survivors of trauma and torture continues to be vigorously debated (Campbell, 2007; Johnson \& Thompson, 2008; Marsella, Friedman, \& Spain, 1992; Nickerson, Bryant, Silove, \& Steel, 2011). Many clinicians providing individual and group interventions recognize these limitations and move beyond the characteristics of the PTSD diagnosis when conducting treatment (Bunn, Goesel, Kinet, \& Ray, 2015). Indeed, Miller and Rassmussen (Miller \& Rasmussen, 2010) suggest that daily stressors and post migration living difficulties (e.g., lack of housing, poverty, language barriers, discrimination) should be prioritized as the initial focus of mental health treatment for refugees. Furthermore, a recent review of existing interventions for survivors of torture identified three overlapping categories of refugee group mental health intervention currently utilized including: 1) supportive (community or group connection); 2) stage one (psychoeducation and symptom reduction); and 3) stage two (trauma narrative) (Bunn et al., 2015). Similarly, in a rigorous review of psychological treatments of PTSD in adult refugees, Nickerson and colleagues (Nickerson et al., 2011) refer to two contrasting approaches; trauma-focused therapy and multimodal interventions (Nickerson et al., 2011). Another recent review of 88 studies of interventions for survivors of torture and systematic violence concluded that although Cognitive Behavior Therapy, including exposure techniques, has the strongest evidence for reducing mental health symptoms associated with PTSD, Depression and Anxiety, they strongly recommended further research and randomized control trials of other promising interventions including multidisciplinary therapies (Weiss et al., 2016). Collectively, these review articles emphasize the need for greater research addressing varying multimodal treatments. Many broader interventions not only address symptoms related to PTSD and trauma, but also attend to post-migration difficulties, daily stressors, bio-psychosocial factors, acculturation, and human rights atrocities (e.g., (Bunn et al., 2015) and (Nickerson et al., 2011); (Weiss 
et al., 2016). A unifying conceptualization for refugee service provision is important when considering scientific investigation of the impact of various interventions on differing outcomes.

As the specialty field of treatment services for refugees and survivors of torture further develops, it is clear that a broad variety of interventions will continue to be provided by clinical communities. Given the complexities of the refugee experience and extensive range of responses, we propose a unifying conceptualization acknowledging the importance of varying clinical interventions. Moreover, the proposed model is intended to foster empirical testing regarding outcomes associated with different treatment modalities. The conceptualization (see Figure 1) is intended to be nonpathologizing, respecting a range of cultural responses (including strengths and resilience), while also acknowledging the ongoing impact of war. The model includes ongoing Chronic Traumatic Stress (CTS) related to war and political conflict and migration, in addition to post migration challenges, and daily stressors. As witnessed by many clinicians, protective and risk factors may moderate the impact of traumatic experiences on the individual embedded in his/her family, community, and culture (Bronfenbrenner, 1992).

Outcomes of these traumatic and stressful events include resilience and wellness, along with mental health and physical problems, and trauma specific symptoms. While the established PTSD diagnosis guides treatment for reducing a specific set of symptoms, the proposed conceptualization expands the understanding of sequelae beyond post-traumatic stress symptoms. In this paper, we first present the utility and limitations of the PTSD diagnosis for refugees and survivors of torture. Next, we present the CTS model outlining the psychological and physical sequelae associated with the broader refugee experience. Finally, utilizing the CTS framework, we highlight the existence of multidisciplinary interventions currently incorporated by practitioners and highlight the need for further research.

\section{Utility and limitations of PTSD emphasis}

The establishment of Post Traumatic Stress Disorder (PTSD) as a clinical diagnosis provided the mental health community with a platform from which clinicians and researchers could collectively develop empirical frameworks for assessment, intervention and prevention. Heretofore, soldiers, victims of rape and survivors of accidents did not have adequate diagnostic or treatment options. The field of traumatic stress flourished with efficacious treatments (e.g., Prolonged Exposure [PE], Cognitive Processing Therapy [CPT], and Trauma Focused Cognitive Behavioral Therapy [TF-CBT] and provided a framework for mental health services (Cohen, Mannarino, \& Deblinger, 2006; Foa \& Rothbaum, 2001; Resick, Monson, \& Chard, 2016; Resick \& Schnicke, 1993). However, the heterogeneity of post-traumatic stress reactions emphasize the problems with assigning a single PTSD diagnosis for the range of responses to trauma (GalatzerLevy \& Bryant, 2013; Herman, 1992). The conceptualizations of Complex-PTSD, Complex Developmental Trauma, and Disorders of Extreme Stress not otherwise specified (DESNOS), collectively attempted to move beyond PTSD as it is delineated by the Diagnostic and Statistical Manual of Mental Disorders (DSM III, DSM IV). These affiliated conceptualizations give 
broader recognition to the complexities involved in diagnosing traumatic stress, however, refugees and survivors of torture present unique experiential and cross cultural considerations not fully accounted for by any of these diagnoses (McFarlane \& Kaplan, 2012). Additionally, while the DSM 5 PTSD diagnosis requires that an external event(s), such as combat or rape has been experienced, an emphasis is placed on the reactions by individuals to these event(s) (Association, 2013). Labeling the human response to war trauma as disordered is problematic, as it shifts blame to the individual and takes the attention off the event(s). This emphasis on pathology implies that there is a correct response and duration of responses to these horrific traumatic events. Moreover, individuals from other cultures may internalize this diagnosis and believe that there is something inherently wrong with them as a result of their experienced trauma.

It has been empirically demonstrated that many refugees and survivors of torture who present with PTSD symptomatology (e.g., flashbacks, intrusive thinking, dissociation), and symptoms associated with depression and anxiety, may sufficiently be treated with trauma focused cognitive behavior therapy treatments with an emphasis on exposure techniques (Nickerson et al., 2011; Weiss et al., 2016). However, as described by a number of authors (Bunn et al., 2015; McFarlane \& Kaplan, 2012; Nickerson et al., 2011; Weiss et al., 2016), further research is needed as refugee mental and physical health needs are complex and include concerns far beyond trauma related symptomatology. As such, clinicians are implementing interventions that incorporate functionality, social support, psychoeducation, and behavioral activation in conjunction with trauma specific techniques and attendance to post migration and daily stressors (Bunn et al., 2015). Unfortunately, many of these interventions are not rigorously empirically tested. However, pre-post testing often shows promising results for many of these comprehensive or interdisciplinary programs (Bunn et al., 2015; Weiss et al., 2016). Based on their recent review, Weiss and colleagues (Weiss et al., 2016) concluded that regardless of the intervention provided, it is critical for clinicians and researchers to collaborate and show that outcomes are moving in the expected direction with either pre-post testing at a minimum or randomized controlled trials, if possible.

\section{Introduction to the Chronic Traumatic Stress (CTS) model}

Although the CTS model is conceptual, it is designed for clinical purposes and has the added benefit of providing guidance for empirical investigation. When studying the effects of traumatic war events and stress on individuals, families and communities, different cultures may have varied psychological and physical outcomes. These refugee population differences need to be taken into account when interpreting symptoms and deciding which outcomes to measure and at what frequency. In the overview presented, events and stressors are described as precipitants, and physical and psychological strengths and challenges are described as outcomes (see Figure 1). Moderating risk and protective factors are exhibited at the individual, family, community, and cultural levels. It is important to note that studies reviewed in the following sections are not comprehensive but rather offered as examples of precipitants, outcomes, and moderators which can be measured using the CTS framework. 
Figure 1: Chronic Traumatic Stress (CTS) Framework

\section{Protective and Risk Factors}

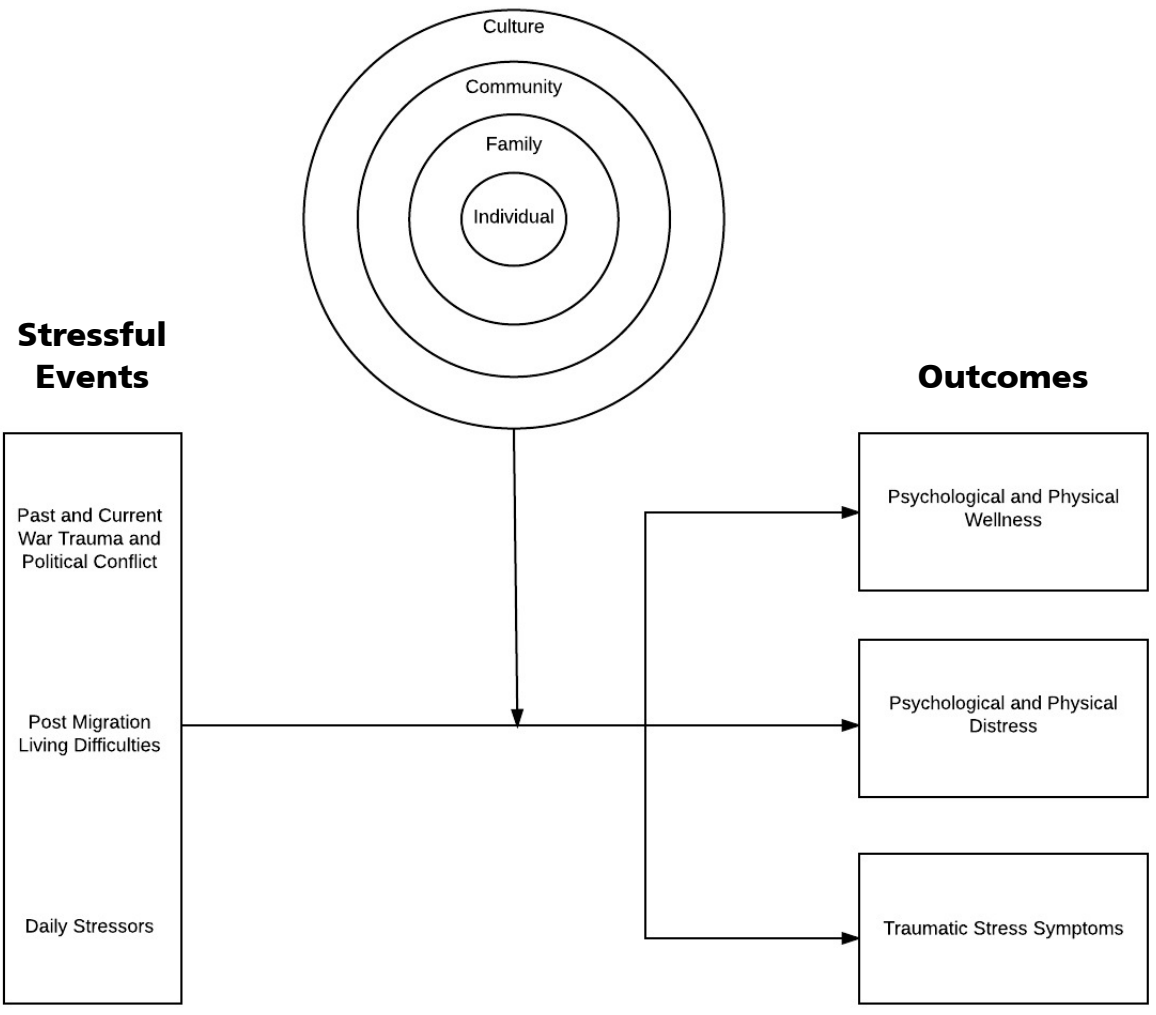

\section{Traumatic and stressful events - precipitants}

As shown in the CTS Framework, refugees and survivors of torture experience a range of traumatic and stressful events, including past and current events. These include Chronic Traumatic Stress (CTS) related to war and political conflict, post migration living difficulties, and daily hassles. These constructs are intentionally separated in the CTS model in order to retain the ability to empirically investigate their unique and shared contribution to outcomes.

\section{Chronic Traumatic Stress (CTS)}

It is well established that refugees experience traumatic stress associated with war and political conflict including physical injury, sexual assault, rape, the witnessing of violence, loss of family members, and torture (Mollica, McDonald, Massagli, \& Silove, 2004; Peel, 2004). In addition to specific 
traumatic events, refugees and survivors of torture may face stressors related to safety, lack of food and shelter, and appropriate medical care while attempting to escape persecution (Fazel et al., 2005). In the CTS model, these war related experiences are characterized as traumatic stress related to "past and current war trauma and political conflict." Due to the ongoing nature of many wars and political conflict, refugees may experience chronic traumatic stress even after they have been resettled (e.g., on-going war or unstable political climate in country of origin).

\section{Post migration living difficulties}

During post migration and resettlement, accompanying stressors may exacerbate the impact of initial traumatic experiences (Miller \& Rasmussen, 2010). The empirical literature indicates that the most prominent post migration stressors among refugee populations include language barriers (Goodkind et al., 2014; Schweitzer, Brough, Vromans, \& Asic-Kobe, 2011), inadequate social support (Goodkind et al., 2014; Teodorescu, Heir, Hauff, Wentzel-Larsen, \& Lien, 2012), and worries about family members who reside in other countries (Schweitzer et al., 2011). Other frequently cited post migration living difficulties are unemployment, poverty, lack of housing, discrimination, acculturation struggles, family problems, and transportation difficulties (Schweitzer et al., 2011). Financial challenges can be particularly stressful as monetary assistance during resettlement is often extremely time limited. Further, discrimination related to varying cultural backgrounds and religious affiliations can exacerbate stress (Perera et al., 2013). Indeed, a number of studies have shown the negative impact of post migration living difficulties on psychological and physical outcomes in refugees (Schweitzer et al., 2011; Silove, Steel, McGorry, \& Mohan, 1998). Including this construct when measuring stress associated with the refugee experience is critical as these stressors may impact treatment outcomes and are sometimes necessary targets of treatment. For example, lack of housing may exacerbate sleep problems which may enhance trauma symptoms. Addressing this challenge may dramatically impact treatment outcomes.

\section{Daily stressors}

Although the terms "daily stressors" and "post migration living difficulties" are often used interchangeably in the literature on refugee stress (Miller \& Rasmussen, 2010), we purposefully separate these constructs in our model. Support for separating these constructs is demonstrated by research with immigrants in which findings showed separate contributions of acculturation specific and acculturation non-specific daily hassles on psychological distress (Lay \& Nguyen, 1998). As previously described, we define post migration difficulties as those challenges typically related to the refugee experience after resettlement in a host country. Alternatively, daily stressors may be independent of the refugee struggle and include hassles commonly experienced by the broader population of refugees and nonrefugees. Events such as managing financial obligations, difficulty finding childcare, or unexpected car troubles are considered daily hassles (Safdar \& Lay, 2003). In our model, these constructs can be measured together or independently to assess the differential impact on well-being and distress.

\section{Protective and risk factors - moderating variables}

Traumatic and stressful life events often impact refugees or survivors of torture at 
the individual level. However, it is well recognized that the impact of trauma and stressful events permeates relationships, families, communities, as well as societies (Nickerson et al., 2011). The ecological model underscores the interaction between individual, family, community, and cultural levels to help describe individual functioning (Bronfenbrenner, 1992). In the CTS framework, the interplay between individuals and surrounding environments is central to understanding the impact of war, post-migration living difficulties and concomitant daily stress. Specifically, protective and risk factors at each level may moderate the effect of stressful and traumatic life events. Risk factors interact with stressful events to increase the likelihood of negative physical and psychological outcomes whereas protective factors mitigate negative outcomes and increase resilience. Empirical investigation of protective and risk factors can assist with the testing and development of effective multimodal interventions.

While resilience is defined differently by various authors (Barber \& Doty, 2013; Hoge, Austin, \& Pollack, 2007), we refer to this construct as positive adaptation in the context of severe adverse circumstances (Luthar, Cicchetti, \& Becker, 2000; Hooberman, Rosenfeld, Rasmussen, \& Keller, 2010). Research has demonstrated that protective factors increasing resilience such as adaptive coping styles, social support, and community engagement may mitigate the negative effects of trauma and reduce psychological impairment (Başoğlu, Paker, Özmen, Taşdemir, \& Şahin, 1994; Bonanno, 2004; Hooberman et al., 2010). Individual protective factors may include genetic predisposition, age, sex, coping style, and emotion regulation skills (Hoge et al., 2007). Protective factors increasing resilience may also be noted in families (e.g., family cohesiveness, family support, economic status), communities (e.g., community resources, community support) and cultures (e.g., spirituality, cultural identity, cultural traditions). Studies addressing the negative impact of risk factors on the functioning of refugees and torture survivors have focused on all levels, including individual risk factors (e.g., substance abuse, suicidal thoughts), familial factors (e.g., domestic violence), community factors (e.g., community violence) and cultural factors (e.g., acculturation difficulties, discrimination, historical loss) (see Fleming \& Ledogar, 2008). The moderating effect of risk and protective factors on the impact of stress across all levels can be empirically measured and may enhance our understanding of the impact of multimodal interventions for refugees.

\section{Outcomes}

The vast majority of studies assessing outcomes associated with refugee trauma focus on the negative impacts including psychological disorders, physical ailments, and trauma symptoms. Within the CTS framework, while negative consequences of traumatic events are highlighted, an emphasis is also placed on understanding psychological and physical well-being in the face of war trauma.

\section{Psychological and physical well-being}

The construct of psychological wellbeing has been described in various ways throughout the literature (e.g., Berry, 1997; Lazarus \& Folkman, 1984; Ryan, Dooley, $\&$ Benson, 2008). While psychological wellbeing within the CTS Framework includes the lack of significant symptoms of anxiety, depression and PTSD, it also includes the utilization of psychological resources (e.g., 
coping mechanisms, distress tolerance techniques, acceptance strategies) and accessing social and community supports (e.g., making decisions according to one's identified values, cultivating a sense of meaning). Additionally, psychological wellbeing includes acceptance and management of symptoms associated with anxiety, depression, and post-traumatic stress. The construct of physical well-being may include healthy sleep patterns, exercise, nutrition, abstaining from substance abuse and management of medical conditions, along with the lack of significant physical ailments. Service providers often witness the amazing psychological and physical strengths of refugees and survivors of torture, however these constructs are rarely measured as outcome variables in treatment studies. The empirical investigation of treatment outcomes associated with increased wellbeing may be an important emphasis for promising multimodal treatments that focus on enhancing strength-based factors.

\section{General psychological distress and physical problems}

Disentangling psychological and physical sequelae of war trauma can be challenging, if not impossible, as conditions within these domains frequently overlap. Nevertheless, the literature is replete with support for the deleterious psychological and physical outcomes of war. Specifically, posttraumatic stress, anxiety and depression are among the most frequently cited psychological symptoms experienced by refugees and survivors of torture (Schubert \& Punamäki, 2011). Sleep disturbances (e.g., insomnia and nightmares) and somatic complaints (e.g., gastrointestinal problems, headaches, dizziness, and chronic pain) have all been empirically demonstrated to be associated with war trauma (Hinton,
Hinton, Eng, \& Choung, 2012; Hinton, Hinton, Pich, Loeum, \& Pollack, 2009; Kirmayer, Groleau, Looper, \& Dao, 2004). Rates of traditionally defined physical ailments such as hypertension, diabetes, and obesity have also been shown to be higher in refugee populations as compared to non-refugees (Bhatta, Shakya, Assad, \& Zullo, 2015). Moreover, although not often empirically tested as outcome variables for intervention, traumatic brain injuries and chronic pain are also common (Weiss et al., 2016). Including physical problems and general psychological distress measures (beyond trauma responses) may broaden our understanding of the impact of the multimodal interventions for refugees.

\section{Traumatic stress symptoms}

Symptoms of PTSD delineated by the DSM 5 include intrusive symptoms (e.g., recurrent memories, flashbacks, nightmares); avoidance of trauma related stimuli; negative alterations in cognitions and mood (e.g., persistent negative beliefs about the world); and alterations in arousal and reactivity (e.g., hypervigilance, exaggerated startle response) (American Psychiatric Association, 2013). Trauma specific outcomes in refugees may be commensurate with symptoms included in the DSM 5 but may also include somatic complaints such as headaches and gastrointestinal problems (Hinton \& Lewis-Fernández, 2011). The traditional symptoms of PTSD as outlined in the western diagnostic system often co-occur with the previously mentioned symptoms of general distress. Additionally, cultural perceptions of mental illness and health may impact interpretation and endorsement of trauma related symptoms. For example, in the Bhutanese population, anxiety driven by fear of distressed ancestors may exacerbate symptoms and add to traumatic stress 
symptoms. Regardless of the intervention provided, it is important that empirical investigation of treatment outcomes for refugees include a measure of traumatic stress symptoms along with general physical and psychological well-being and distress as delineated in the comprehensive CTS model.

\section{Summary}

Treatment modalities and intervention planning depends on theoretical perspectives regarding the etiology and maintenance of impairment. Existing treatments to address trauma symptoms experienced by refugees and survivors of torture are documented in the literature and utilized globally (e.g., Prolonged Exposure (PE) (Foa \& Rothbaum, 2001); Cognitive Processing Therapy (CPT) (Resick \& Schnicke, 1993; Schulz, Resick, Huber, \& Griffin, 2006); Narrative Exposure Therapy (NET) (Schauer, Neuner, \& Elbert, 2005); Culturally Adapted-Cognitive Behavioral Therapy (CA-CBT) (Hinton, Rivera, Hofmann, Barlow, \& Otto, 2012). Additionally, cultural adaptations to existing empirically based interventions have been developed and utilized e.g., (Bunn et al., 2015). Finally, novel, multimodal, interdisciplinary, and comprehensive treatments are utilized in clinics and treatment centers around the world, however, these treatments rarely receive empirical investigation. Together, these interventions address individual symptoms related to PTSD and trauma, and stressors associated with post-migration difficulties and daily hassles, in addition to attending to family, community, and cultural factors. Our proposed conceptualization attempts to provide guidance for interdisciplinary treatments, including medical, social, and psychological treatments (e.g, supportive, coping technique-based, and exposure- based treatments) (Bunn et al., 2015). Additionally, empirical testing can be conducted regarding outcomes associated with existing interdisciplinary treatments.

\section{CTA Framework guides empirical investigation}

The CTS Framework guides empirical investigation beyond a focus on PTSD and associated symptoms by providing a comprehensive, strength based model. Throughout this article, we emphasize the significance of all modalities of biopsychosocial treatment; 1) supportive (community or group connection); 2) psychoeducation and symptom reduction; 3) addressing the trauma narrative; (Bunn et al., 2015) and 4) attention to physical health. While intervention focused solely on PTSD assesses the occurrence of a past event (or events), the CTS model highlights chronic stress including ongoing traumatic stress events related to war, post migration stress, and daily hassles in order to assess the unique and combined impact of these events. Also, the CTS moderating variables at all ecological levels emphasize the need to investigate risk and protective factors that can be addressed during intervention and may have a unique impact on outcomes. Finally, the outcome section of the model exhibits the importance of assessing well-being, resilience, and strengths, along with physical and psychological sequelae. PTSD interventions typically target the reduction of trauma symptoms as the sole outcome measure.

Further empirical testing in the form of randomized control trials would benefit the field regarding the assessment of comprehensive treatments for refugees and survivors of torture. Unfortunately, conducting randomized control trials can be costly, requiring grant funding and advanced 
statistical support. While randomized control trials represent the gold standard, this may not be feasible for many or most programs in which this cost may be prohibitive. While practitioners are devoted and believe strongly in the effectiveness of their services, without measurement of program success, these methods remain understudied. We suggest that measurement conducted before, during, and after treatment may be feasible for programs and important to understanding the impact of interdisciplinary intervention (Weiss et al., 2016).

\section{Measurement-based care for interdisciplinary interventions}

Assessment conducted prior to, during, and after, interdisciplinary intervention is often not conducted by practitioners. For those programs that do collect data for clinical or program evaluation purposes, results are rarely published or widely disseminated. Measurement can provide knowledge regarding overall program success, useful program modifications, and may enhance future funding opportunities. In this paper, we provide a model that can be used as guidance for measurement of treatment variables and clinical outcomes. We suggest that regardless of the combination of treatment domains (e.g., social, physical, psychological), it is beneficial to include a comprehensive stress profile as explicated in the CTS model (e.g., past and current war trauma and political conflict, post migration living difficulties and daily stress). Such measurement provides information regarding the separate and additive impact of stress upon the participants prior to, during, and post intervention. Moreover, when assessing the impact of the intervention, adding an analysis of potential moderating variables to the empirical investigation such as protective factors (e.g., family and/ or community support, economic status, and spirituality) may shed further light on important variables related to change. For example, a program may enhance healthy psychological well-being through community engagement and behavioral activation while not necessarily impacting the experience of sadness associated with the loss of family members or anxiety associated with fear of being deported. Finally, adding outcome measures that go beyond the intended focus of the intervention may demonstrate the broader impact of interdisciplinary treatments. For example, trauma specific measures can be used as outcomes for interventions aimed at reducing social stress (e.g., poverty, unemployment). Although not commonly measured when social work interventions are employed, it is possible that traumatic stress symptoms (e.g., flashbacks, hypervigilance, avoidance) may decrease after an intervention targeting outcomes such as secure housing or gainful employment is conducted. Similarly, measures regarding chronic pain or other physical health variables (e.g., hypertension) could be added as outcome measures for treatment intended to target trauma symptoms. The possibility that one treatment domain (e.g., physical, psychological, or social) may impact another domain is central to outcome measurement as delineated in the CTS model. Finally, collaborative efforts, such as those demonstrated by the National Consortium of Torture Treatment Programs (NCTTP), to disseminate cumulative data collected across multiple programs would be beneficial to the broader community of service providers by increasing dissemination of novel evidence-based approaches. 


\section{References}

American Psychiatric Association, (2013). Diagnostic and statistical manual of mental disorders (DSM$5 \circledR)$ : American Psychiatric Pub.

Barber, B. K., \& Doty, S. B. (2013). How can a majority be resilient? Critiquing the utility of the construct of resilience through a focus on youth in contexts of political conflict Handbook of Resilience in Children of War (pp. 233-252). Springer.

Başoğlu, M., Paker, M., Özmen, E., Taşdemir, Ö., \& Şahin, D. (1994). Factors related to long-term traumatic stress responses in survivors of torture in Turkey. $\mathscr{F} A M A, 272(5), 357-363$.

Berry, J. W. (1997). Immigration, Acculturation, and Adaptation. Applied Psychology 46 (1) 1997, 5-34. doi.org/10.1111/j.1464-0597.1997.tb01087.x

Bhatta, M. P., Shakya, S., Assad, L., \& Zullo, M. D. (2015). Chronic Disease Burden Among Bhutanese Refugee Women Aged 18-65 Years Resettled in Northeast Ohio, United States, 2008-2011. F Immigr Minor Health, 17(4), 1169 1176. doi:10.1007/s10903-014-0040-9

Bonanno, G. A. (2004). Loss, trauma, and human resilience: have we underestimated the human capacity to thrive after extremely aversive events? American Psychologist, 59(1), 20.

Bronfenbrenner, U. (1992). Ecological systems theory. Jessica Kingsley Publishers.

Bunn, M., Goesel, C., Kinet, M., \& Ray, F. (2015). Group treatment for survivors of torture and severe violence: A literature review. Torture 26(1), 45.

Campbell, T. A. (2007). Psychological assessment, diagnosis, and treatment of torture survivors: A review. Clinical Psychology Review, 27(5), 628-641. doi:http://dx.doi.org/10.1016/j. cpr.2007.02.003

Cohen, J. A., Mannarino, A. P., \& Deblinger, E. (2006). Treating trauma and traumatic grief in children and adolescents. Guilford Press.

Fazel, M., Wheeler, J., \& Danesh, J. (2005). Prevalence of serious mental disorder in 7000 refugees resettled in western countries: a systematic review. The Lancet, 365(9467), 13091314. doi:10.1016/S0140-6736(05)61027-6

Fleming, J., \& Ledogar, R. J. (2008). Resilience, an evolving concept: A review of literature relevant to Aboriginal research. Pimatisizin, 6(2), 7 .

Foa, E. B., \& Rothbaum, B. O. (2001). Treating the trauma of rape: Cognitive-behavioral therapy for PTSD: Guilford Press.

Galatzer-Levy, I. R., \& Bryant, R. A. (2013). 636,120 ways to have posttraumatic stress disorder. Perspectives on Psychological Science, 8(6), 651-662.
Goodkind, J. R., Hess, J. M., Isakson, B., LaNoue, M., Githinji, A., Roche, N., . . Parker, D. P. (2014). Reducing refugee mental health disparities: A community-based intervention to address postmigration stressors with African adults. Psychological Services, 11(3), 333.

Herman, J. L. (1992). Complex PTSD: A syndrome in survivors of prolonged and repeated trauma. fournal of Traumatic Stress, 5(3), 377-391.

Hinton, D. E., Hinton, A. L., Eng, K. T., \& Choung, S. (2012). PTSD and key somatic complaints and cultural syndromes among rural Cambodians: The results of a needs assessment survey. Medical Anthropology Quarterly, 26(3), 383-407.

Hinton, D. E., Hinton, A. L., Pich, V., Loeum, J., \& Pollack, M. H. (2009). Nightmares among Cambodian refugees: The breaching of concentric ontological security. Culture, Medicine, and Psychiatry, 33(2), 219-265.

Hinton, D. E., \& Lewis-Fernández, R. (2011). The cross-cultural validity of posttraumatic stress disorder: implications for DSM-5. Depression and Anxiety, 28(9), 783-801.

Hinton, D. E., Rivera, E. I., Hofmann, S. G., Barlow, D. H., \& Otto, M.W. (2012). Adapting CBT for traumatized refugees and ethnic minority patients: examples from culturally adapted CBT (CA-CBT). Transcultural Psychiatry, 49(2), 340-365.

Hoge, E. A., Austin, E. D., \& Pollack, M. H. (2007). Resilience: research evidence and conceptual considerations for posttraumatic stress disorder. Depression and Anxiety, 24(2), 139-152.

Hooberman, J., Rosenfeld, B., Rasmussen, A., \& Keller, A. (2010). Resilience in trauma-exposed refugees: The moderating effect of coping style on resilience variables. American fournal of Orthopsychiatry, 80(4), 557.

Johnson, H., \& Thompson, A. (2008). The development and maintenance of post-traumatic stress disorder (PTSD) in civilian adult survivors of war trauma and torture: A review. Clinical Psychology Review, 28(1), 36-47. doi:http://dx.doi. org/10.1016/j.cpr.2007.01.017

Kirmayer, L. J., Groleau, D., Looper, K. J., \& Dao, M. D. (2004). Explaining medically unexplained symptoms. The Canadian fournal of Psychiatry, 49(10), 663-672.

Lay, C., \& Nguyen, T. (1998). The role of acculturation-related and acculturation nonspecific daily hassles: Vietnamese-Canadian students and psychological distress. Canadian fournal of Behavioural Science/Revue canadienne des sciences du comportement, 30(3), 172. 
Lazarus, R. S., \& Folkman, S. (1984). Coping and adaptation. The Handbook of Behavioral Medicine, 282-325.

Luthar, S. S., Cicchetti, D., \& Becker, B. (2000). The construct of resilience: A critical evaluation and guidelines for future work. Child development, 71(3), 543-562.

Marsella, A., Friedman, M., \& Spain, E. H. (1992). A selective review of the literature on ethnocultural aspects of PTSD. PTSD Research Quarterly, 3(2), 1-7.

McFarlane, C. A., \& Kaplan, I. (2012). Evidencebased psychological interventions for adult survivors of torture and trauma: a 30-year review. Transcultural psychiatry, 49(3-4), 539-567.

Miller, K. E., \& Rasmussen, A. (2010). War exposure, daily stressors, and mental health in conflict and post-conflict settings: bridging the divide between trauma-focused and psychosocial frameworks. Social science \& medicine, 70(1), 7-16.

Mollica, R. F., McDonald, L., Massagli, M., \& Silove, D. M. (2004). Measuring trauma, measuring torture. Cambridge, MA: Harvard University.

Nickerson, A., Bryant, R. A., Silove, D., \& Steel, Z. (2011). A critical review of psychological treatments of posttraumatic stress disorder in refugees. Clinical Psychology Review, 31(3), 399-417.

Peel, M. (2004). Rape as a Method of Torture. Medical Foundation for the Care of Victims of Torture.

Perera, S., Gavian, M., Frazier, P., Johnson, D., Spring, M., Westermeyer, J., Butcher, J., Halcon, L., Robertson, C., Savik, K., Jaranson, J. (2013). A longitudinal study of demographic factors associated with stressors and symptoms in African refugees. American fournal of Orthopsychiatry, 83(4), 472-482.

Porter, M., \& Haslam, N. (2005). Predisplacement and postdisplacement factors associated with mental health of refugees and internally displaced persons: a meta-analysis. $\mathcal{F} A M A, 294(5), 602-612$.

Resick, P. A., Monson, C. M., \& Chard, K. M. (2016). Cognitive Processing Therapy for PTSD: A Comprehensive Manual. Guilford Publications.

Resick, P. A., \& Schnicke, M. (1993). Cognitive processing therapy for rape victims: A treatment manual (Vol. 4). Sage.

Ryan, D., Dooley, B., \& Benson, C. (2008). Theoretical perspectives on post-migration adaptation and psychological well-being among refugees: Towards a resource-based model. fournal of Refugee Studies, 21(1), 1-18.

Safdar, S. F., \& Lay, C. H. (2003). The Relations of Immigrant-Specific and Immigrant-Nonspecific Daily Hassles to Distress Controlling for Psychological Adjustment and Cultural
Competence. Fournal of Applied Social Psychology, 33(2), 299-320.

Schauer, M., Neuner, F., \& Elbert, T. (2005).

Narrative exposure therapy: $A$ short-term intervention for traumatic stress disorders after war, terror, or torture. Hogrefe \& Huber Publishers.

Schubert, C. C., \& Punamäki, R.-L. (2011). Mental health among torture survivors: cultural background, refugee status and gender. Nordic fournal of Psychiatry, 65(3), 175-182.

Schulz, P. M., Resick, P. A., Huber, L. C., \& Griffin, M. G. (2006). The effectiveness of cognitive processing therapy for PTSD with refugees in a community setting. Cognitive and Behavioral Practice, 13(4), 322-331.

Schweitzer, R. D., Brough, M., Vromans, L., \& AsicKobe, M. (2011). Mental health of newly arrived Burmese refugees in Australia: contributions of pre-migration and post-migration experience. Australian and New Zealand fournal of Psychiatry, 45(4), 299-307.

Silove, D., Steel, Z., McGorry, P., \& Mohan, P. (1998). Trauma exposure, postmigration stressors, and symptoms of anxiety, depression and post-traumatic stress in Tamil asylumseekers: comparison with refugees and immigrants. Acta Psychiatrica Scandinavica, 97(3), 175-181.

Steel, Z., Chey, T., Silove, D., Marnane, C., Bryant, R. A., \& Van Ommeren, M. (2009). Association of torture and other potentially traumatic events with mental health outcomes among populations exposed to mass conflict and displacement: a systematic review and meta-analysis. $\mathcal{F} A M A$, 302(5), 537-549.

Teodorescu, D. S., Heir, T., Hauff, E., WentzelLarsen, T., \& Lien, L. (2012). Mental health problems and post-migration stress among multitraumatized refugees attending outpatient clinics upon resettlement to Norway. Scandinavian fournal of Psychology, 53(4), 316-332.

UNHCR. (2018). UNHCR Global Trends

Weiss, W. M., Ugueto, A. M., Mahmooth, Z., Murray, L. K., Hall, B. J., Nadison, M., Rasmussen, A., Lee, J. S., Vazzano, A., Bass, J. \& Bolton, P. (2016). Mental health interventions and priorities for research for adult survivors of torture and systematic violence: a review of the literature. Torture, 26, 17-44. 